\title{
Depth and combined infection is important predictor of lower extremity amputations in hospitalized diabetic foot ulcer patients
}

\author{
Eun-Gyo Jeong ${ }^{1}$, Sung Shim Cho ${ }^{1}$, Sang-Hoon Lee ${ }^{1}$, Kang-Min Lee ${ }^{1}$, Seo-Kyung Woo ${ }^{1}$, Yoongoo Kang ${ }^{1}$, \\ Jae-Seung Yun ${ }^{1}$, Seon-Ah Cha ${ }^{1}$, Yoon-Jung Kim² ${ }^{2}$, Yu-Bae Ahn ${ }^{1}$, Seung-Hyun $\mathrm{Ko}^{1}$, and Jung-Min Lee ${ }^{3}$
}

\begin{abstract}
${ }^{1}$ Division of Endocrinology and Metabolism, Department of Internal Medicine, ${ }^{2}$ Department of Orthopedic Surgery, College of Medicine, St. Vincent's Hospital, The Catholic University of Korea, Suwon; ${ }^{3}$ Division of Endocrinology and Metabolism, Department of Internal Medicine, College of Medicine, St. Paul's Hospital, The Catholic University of Korea, Seoul, Korea
\end{abstract}

\author{
Received: May 23, 2016 \\ Revised : August 15, 2016 \\ Accepted: October 6, 2016

\section{Correspondence to} \\ Jung-Min Lee, M.D. \\ Division of Endocrinology and \\ Metabolism, Department of \\ Internal Medicine, College of \\ Medicine, St. Paul's Hospital, The \\ Catholic University of Korea, 180 \\ Wangsan-ro, Dongdaemun-gu, \\ Seoul 02559, Korea \\ Tel: +82-2-958-2379 \\ Fax: +82-2-968-7250 \\ E-mail: leejm68@catholic.ac.kr
}

\begin{abstract}
Background/Aims: As the prevalence of diabetes mellitus and its complications increase rapidly, diabetic foot ulcers (DFUs), which are a major diabetic complication, are expected to increase. For prevention and effective treatment, it is important to understand the clinical course of DFUs. The aim of this study was to investigate the natural course and predictors of amputation in patients with DFUs who required hospitalization

Methods: A total of 209 patients with type 2 diabetes, aged 30 to 85 years, who visited emergency department or needed hospitalization due to DFUs were consecutively enrolled from May 2012 to January 2016, by retrospective medical record review. The main outcome was lower extremity amputation (LEA).

Results: Among 192 patients who completed follow-up, 113 patients (58.9\%) required LEAs. Compared to patients without amputation, baseline levels of white blood cell counts and C-reactive protein were higher in patients with amputation. In addition, bone and joint involvement was more frequently observed in patients with amputation. Multivariable regression analysis revealed that combined infection (odds ratio [OR], 11.39; 95\% confidence interval [CI], 2.55 to 50.93; $p=0.001$ ) and bone or joint involvement (OR, 3.74; 95\% CI, 1.10 to 12.70 ; $p=0.035$ ) were significantly associated with an increased risk of LEA.

Conclusions: The depth of the wound and combined infection of DFU, rather than the extent of the wound, were significant prognostic factors of LEAs in patients with type 2 diabetes.
\end{abstract}

Keywords: Diabetes mellitus; Diabetic foot; Ulcer; Prognosis; Amputation

\section{INTRODUCTION}

The prevalence of type 2 diabetes is rapidly increasing. In 2012, diagnosed and undiagnosed diabetes among people aged 20 years or older was $12.3 \%$ in the United States [1]. The World Health Organization reported that the global prevalence of diabetes in adults over 18 years in 2014 was estimated to be $9 \%$ [2], and the estimated number of patients with diabetes worldwide was reported as 415 million in 2015 [3]. In Korea, according to the Korean National Health and Nutrition Examination Survey 2011 , the prevalence of diabetes in Korea was $10.5 \%$ in adults over 30 years or older [4]. As the prevalence of diabetes mellitus (DM) and its complications increase rapidly, the incidence of diabetic foot ulcer (DFU) is also expected to increase [5]. According to the data from ' $\mathrm{Di}$ - 
abetes in Korea 2007,' the $44.8 \%$ of the foot amputated patients in 2003 had diabetes [6]. Patients with diabetes were 10.1 times more likely to undergo foot amputation, and 7.8 times more likely to have foot ulcer [6]. In addition, about 73,000 non-traumatic lower-limb amputations were performed in adults aged 20 years or older with diagnosed diabetes in the United States in 2010 [1].

DFU is associated with a significant portion of admission, medical costs, disability, and mortality, and is the leading cause of non-traumatic lower extremity amputation (LEA) $[7,8]$. According to one study performed in Korea from December 1994 through December 2002, total medical costs (per capita) of foot amputation and foot ulcer in patients with diabetes were 2.0 and 1.7 times higher, respectively, than those of non-diabetic patients [9]. Mean hospital stay of foot amputation and DFU were 1.6 and 1.3 times longer, respectively, than those of non-diabetic patients [9]. Considering this increasing prevalence of diabetic vascular complications and its related health burden, early detection and prompt management of DFU are urgently required. In addition, for prevention and effective treatment of DFU, it is important to understand the risk factors and clinical course of DFU in patients with type 2 diabetes.

This retrospective cohort study aimed to investigate the predictors of LEA in patients with type 2 diabetes hospitalized for DFU.

\section{METHODS}

\section{Patients}

A total of 380 patients with type 2 diabetes, aged 30 to 85 years, who visited St. Vincent's Hospital in Korea for DFU management were recruited consecutively from May 2012 to January 2016 by retrospective medical record review. Among these patients, 229 subjects who visited the Emergency Department or needed hospitalization for DFU care were included. Twenty patients with type $1 \mathrm{DM}$, gestational DM, or any severe illness, such as liver cirrhosis, heart failure, and malignancy were excluded from the study. The patients with too little available clinical data from the medical record were also excluded (Fig. 1). The Catholic Medical Center Ethics Committee and the Institutional Review Board (IRB No: VC15OISIo207) approved this study. Written informed consents were obtained.

Information about the clinical and demographic characteristics was collected by careful medical record review. Hypertension was defined as systolic blood pressures $\geq$ $140 \mathrm{mmHg}$, diastolic blood pressures $\geq 90 \mathrm{mmHg}$, or the use of antihypertensive medications. Fasting and postprandial plasma glucose levels were measured using an automated enzymatic method (model $7600-$ 110, Hitachi, Tokyo, Japan), and glycated hemoglobin (HbA1c) levels were measured using high-performance liquid chromatography with a reference range of $4.4 \%$ to $6.4 \%$ (Bio-Rad, Montreal, QC, Canada). Total cholesterol, triglycerides, low density lipoprotein, and high density lipoprotein cholesterol were measured enzymatically using an automatic analyzer (model 7600-110). Estimated glomerular filtration rate (eGFR) was assessed using the 4-component Modification of Diet in Renal Disease equation [10]. Baseline white blood cell (WBC) counts and C-reactive protein (CRP) were also measured. We defined smoking status as current or past smokers. Coronary artery disease was defined as a history of diag-

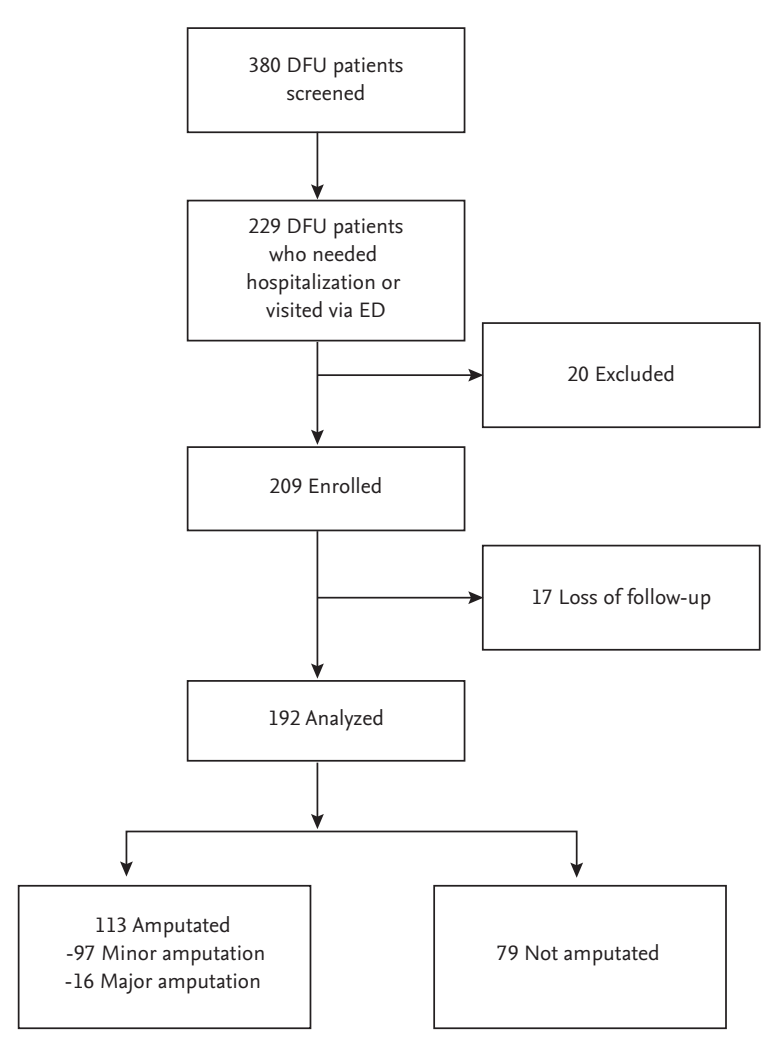

Figure 1. Study flow diagram. DFU, diabetic foot ulcer; ED, Emergency Department. 
nosed angina pectoris by coronary artery angiography, myocardial infarction, or coronary revascularization (coronary bypass surgery or coronary angioplasty) [11]. Stroke history included previous transient ischemic attack or cerebral infarction [11]. Diabetic retinopathy was defined as non-proliferating diabetic retinopathy of any severity and proliferating diabetic retinopathy, which was confirmed by ophthalmologist. Peripheral artery disease (PAD) was defined as ankle-brachial index (ABI) $\leq 0.9$ or compatible findings of $\mathrm{PAD}$ in peripheral arterial angiography or computed tomography (CT).

\section{DFU outcome evaluation}

We classified the DFU wounds according to extent, depth, and severity of infection of each wound at initial visit. When patient had multiple DFUs, the most significant ulcer was selected as the wound for assessment. The extent of the wound was estimated by multiplying the largest diameter by the second largest diameter measured perpendicular to the first diameter. We classified ulcers into one of the following groups: $<10,10$ to 25 , and $\geq 25 \mathrm{~cm}^{2}$. We further classified ulcers by depth: superficial soft tissue, fascia/muscle/tendon level, and bone or joint level. An infected ulcer was defined as a wound with loss of epithelial continuity of overlying skin with both physical findings, such as pus or redness, heat sensation, fluctuation of surrounding tissue (which suggests fluid accumulation) [5], and one of the following signs or abnormal laboratory findings: fever, WBC $\geq 10 \times 10^{9} / \mathrm{L}$ (normal reference range, 4.0 to $\left.10.0 \times 10^{9} / \mathrm{L}\right)$, or $\mathrm{CRP} \geq 0.50$ (normal reference range, 0.01 to $0.47 \mathrm{mg} / \mathrm{dL}$ ). All of the wounds were classified according to the University of Texas classification [5]. The main outcome in this study was LEA, including minor and major amputations. Minor amputation included toe, ray, transmetatarsal, and below-knee amputation. Major amputation was defined as above-knee amputation [12]. We also collected data on hospitalization days and deaths related to DFUs.

\section{Management protocol for DFU and indications for amputations}

When the DFU patients initially visited our hospital, the multidisciplinary team including an orthopedist or plastic surgeon, vascular surgeon, infectious disease specialists, and an endocrinologist decided the management of the DFU. After initial debridement, an as- sessment of the wound was performed. If the orthopedist or plastic surgeon decided that the wounds did not indicate a need for an emergency amputation, studies for the state of blood supply for the wound (CT angiography for peripheral arteries or arteriography) were done. And if the sign of infection was combined with the DFU, wound culture and administration of the empirical antibiotics (in most cases, 3rd line cephalosporins) were performed. Additionally, when extensive muscle necrosis or osteomyelitis was suspected, we considered the magnetic resonance imaging. After completion of all the above evaluation, the team determined whether to amputate the wound or not.

The followings are the indications for amputation at our hospital: (1) infection: life-threatening sepsis or septic shock; (2) blood supply to the tissues: severe peripheral arterial disease (impossible revascularization); and (3) other: extensive muscle necrosis. However, if the surgeon decides that the wound will heal, only debridement can be done to an ulcer which only involves a toe or part of a foot.

\section{Statistical analysis}

The statistical analyses were performed using SPSS version 13.0 (SPSS Inc., Chicago, IL, USA). Continuous variables were presented as the mean $\pm \mathrm{SD}$ values or median (interquartile range), and categorical variables were presented as percentages. Continuous variables were compared with independent Student $t$ tests, while categorical variables were compared using a chi-square tests and Fisher exact tests. Age, sex, smoking history, duration of DM, hypertension, prior amputation history, WBC, CRP, eGFR, oral anti-diabetic medication, antiplatelet agents, statin, presence of PAD, the depth and size of the wounds, and combined infection were included in the univariable analysis. Because there were not enough medical records about whether patients use insulin, the percentage of missing data regarding insulin use was $61.7 \%$, which has potential for bias. Therefore, we excluded insulin use in statistical analyses. The predictive factors were analyzed using the multivariable logistic regression method, including those that were statistically significant in the univariable analysis. A $p<$ 0.05 was considered to be statistically significant. The results were reported as odds ratios (ORs) with 95\% confidence intervals (CIs). 
Table 1. Baseline characteristics of study participants

\begin{tabular}{|c|c|c|c|}
\hline Characteristic & Non-LEA $(\mathrm{n}=79)$ & $\operatorname{LEA}(n=113)$ & $p$ value \\
\hline Male sex & 25.0 & $37 \cdot 4$ & $0.395^{\mathrm{a}}$ \\
\hline Age, yr & $61.8 \pm 13.9$ & $62.8 \pm 12.5$ & $0.616^{b}$ \\
\hline $\mathrm{BMI}, \mathrm{kg} / \mathrm{m}^{2}$ & $24.2 \pm 3.7$ & $23.5 \pm 3.1$ & $0.284^{b}$ \\
\hline Hospital days, day & $19.0(10.0-31.0)$ & $26.0(18.0-38.0)$ & $<0.001^{\mathrm{c}}$ \\
\hline Duration of DM, yr & $16.2 \pm 10.3$ & $16.0 \pm 10.7$ & $0.141^{b}$ \\
\hline Smoking & 31.6 & $27 \cdot 4$ & $0.355^{\mathrm{a}}$ \\
\hline \multicolumn{4}{|l|}{ Comorbidities } \\
\hline Hypertension & 21.9 & 22.4 & $0.294^{\mathrm{a}}$ \\
\hline ESRD & $5 \cdot 7$ & 7.0 & $0.375^{\mathrm{a}}$ \\
\hline Stroke & $4 \cdot 7$ & 7.8 & $0.423^{\mathrm{a}}$ \\
\hline CAD & 3.1 & 6.8 & $0.271^{\mathrm{a}}$ \\
\hline Diabetic retinopathy & 14.6 & 13.0 & $0.335^{\mathrm{a}}$ \\
\hline \multicolumn{4}{|l|}{ Laboratory findings } \\
\hline WBC count, $\times 10^{9} / \mathrm{L}$ & $9 \cdot 7 \pm 4.3$ & $12.0 \pm 5.9$ & $0.004^{b}$ \\
\hline CRP, mg/dL & $4.7 \pm 6.8$ & $8.2 \pm 8.0$ & $0.002^{b}$ \\
\hline Serum creatinine, mg/dL & $1.1(0.85-1.9)$ & $1.00(0.8-1.7)$ & $0.539^{\mathrm{a}}$ \\
\hline $\mathrm{eGFR}, \mathrm{mL} / \mathrm{min} / 1.73 \mathrm{~m}^{2}$ & $54.6(35.3-83.2)$ & $64.0(38.4-87.0)$ & $0.536^{\mathrm{a}}$ \\
\hline Fasting plasma glucose, $\mathrm{mg} / \mathrm{dL}$ & $160.3 \pm 78.2$ & $184.2 \pm 74.1$ & $0.214^{b}$ \\
\hline Baseline HbAıc, \% & $8.9 \pm 2.3$ & $9.0 \pm 2.3$ & $0.675^{\mathrm{b}}$ \\
\hline Total cholesterol, mg/dL & $161.6 \pm 54.6$ & $159.2 \pm 52.4$ & $0.794^{b}$ \\
\hline Triglyceride, mg/dL & $141.4 \pm 89.0$ & $121.8 \pm 72.0$ & $0.165^{b}$ \\
\hline HDL-C, mg/dL & $35.8 \pm 11.4$ & $32.2 \pm 10.3$ & $0.058^{b}$ \\
\hline LDL-C, mg/dL & $95 \cdot 3 \pm 33.1$ & $95.7 \pm 40.4$ & $0.981^{b}$ \\
\hline $\mathrm{ABI}$ & $0.97 \pm 0.27$ & $0.95 \pm 0.27$ & $0.650^{b}$ \\
\hline \multicolumn{4}{|l|}{ Medications } \\
\hline Oral anti-diabetic medications & 23.5 & 34.9 & $0.240^{\mathrm{a}}$ \\
\hline Antiplatelet agents & 14.6 & 20.3 & $0.514^{\mathrm{a}}$ \\
\hline Statin & $13 \cdot 5$ & 15.6 & $0.297^{\mathrm{a}}$ \\
\hline
\end{tabular}

Values are presented as percentage, mean \pm SD, or median (interquartile range). $p<0.05$ was considered to be statistically significant. LEA, lower extremity amputation; BMI, body mass index; DM, diabetes mellitus; ESRD, end-stage renal disease; CAD, coronary artery disease; WBC, white blood cell; CRP, C-reactive protein; eGFR, estimated glomerular filtration rate; HbA1c, glycated hemoglobin; HDL-C, high density lipoprotein cholesterol; LDL-C, low density lipoprotein cholesterol; ABI, ankle-brachial index.

${ }^{\mathrm{a}} \mathrm{Chi}$-square tests and Fisher exact tests is used.

${ }^{\mathrm{b}}$ Independent Student $t$ tests is used.

${ }^{\mathrm{c}}$ Mann-Whitney test is used.

\section{RESULTS}

\section{Baseline characteristics of the study population}

A total of 209 patients (131 males [62.7\%], 78 females [37.3\%]) were included in the study. After excluding 17 patients $(8.1 \%)$ who were lost to follow-up, 192 patients were analyzed.

Mean age was $62.7 \pm 13.3$ years, and mean duration of diabetes was $16.1 \pm 10.5$ years, respectively. Median hospitalization days was 23.0 days (interquartile range, 14.0 to 34.0 ) (Table 1).

During the follow-up, 38 patients (19.8\%) needed re- 
Table 2. Characteristics of diabetes mellitus foot ulcer and clinical course

\begin{tabular}{|c|c|c|c|}
\hline Characteristic & Non-LEA $(\mathrm{n}=79)$ & $\operatorname{LEA}(n=113)$ & $p$ value \\
\hline Deformity & 4.2 & 3.6 & $0.222^{\mathrm{a}}$ \\
\hline Trauma history & $9 \cdot 9$ & $17 \cdot 7$ & $0.265^{\mathrm{a}}$ \\
\hline Prior amputation history & $3 \cdot 7$ & 6.2 & $0.808^{\mathrm{a}}$ \\
\hline Location of DFU & & & $<0.001^{\mathrm{a}}$ \\
\hline Planta & 3.1 & 2.6 & \\
\hline Dorsum & $4 \cdot 7$ & 3.6 & \\
\hline Toe & 18.8 & 45.8 & \\
\hline Others & 14.6 & 6.8 & \\
\hline Combined PAD & 18.2 & 36.0 & $0.003^{\mathrm{a}}$ \\
\hline Extent of DFU, $\mathrm{cm}^{2}$ & & & $0.059^{\mathrm{a}}$ \\
\hline$<10$ & 16.1 & 14.1 & \\
\hline 10 to $<25$ & 8.4 & $7 \cdot 3$ & \\
\hline$\geq 25$ & 3.7 & $9 \cdot 9$ & \\
\hline Bone or joint involvement of DFU & 6.8 & 26.6 & $<0.001^{\mathrm{a}}$ \\
\hline Infection of DFU & $19 \cdot 3$ & $55 \cdot 7$ & $<0.001^{\mathrm{a}}$ \\
\hline Severity of stenosis of peripheral arteries of lower limbs ${ }^{b}$ & & & $0.405^{\mathrm{a}}$ \\
\hline Mild & 2.1 & 2.1 & \\
\hline Moderate & 2.1 & 6.3 & \\
\hline Severe & 6.3 & 20.8 & \\
\hline Angioplasty during whole follow-up & 10.9 & 13.0 & $0.493^{\mathrm{a}}$ \\
\hline Mean HbAic during the follow-up & $8.6 \pm 2.1$ & $8.4 \pm 1.8$ & $0.133^{c}$ \\
\hline Re-hospitalization & 6.8 & 11.0 & $0.470^{\mathrm{a}}$ \\
\hline Death & 0.5 & 2.1 & $1.000^{\mathrm{d}}$ \\
\hline
\end{tabular}

Values are presented as percentage or mean \pm SD. $p<0.05$ was considered to be statistically significant.

LEA, lower extremity amputation; DFU, diabetic foot ulcer; PAD, peripheral artery disease; HbA1c, glycated hemoglobin.

${ }^{a}$ Chi-square tests and Fisher exact tests is used.

${ }^{b}$ Findings from computed tomography angiography or percutaneous peripheral angiography. We classified the severity of stenosis of any arteries that perfused the DFU area as mild, moderate, and severe, which were confirmed by the expert radiologist or vascular surgeons.

${ }^{\mathrm{c}}$ Independent Student $t$ tests is used.

${ }^{\mathrm{d}}$ Mann-Whitney test is used.

hospitalization because of worsening of initial DFU lesion or newly developed DFU (Table 2). Five enrolled patients (2.6\%) died during hospitalization (four patients died from sepsis and one patient died from acute myocardial infarction).

Among this population, 113 patients (58.9\%) required amputations (97 minor and 16 major amputations). Baseline levels of WBC counts and CRP were higher in the LEA group than those of the non-LEA group. However, there were no significant differences in duration of diabetes, presence of end-stage renal disease, cardiovas- cular disease, glycemic control status, lipid profile, and use of antiplatelet agents between LEA and non-LEA groups (Table 1).

\section{Comparison between the group with LEA and non- LEA}

Median hospital days were longer in the LEA group than in the non-LEA group ( 26 days vs. 19 days, $p<0.001$ ) (Table 1). There was no statistical difference in prior amputation history or deformity between LEA and non-LEA group. Also, there was no statistical difference in the 
Table 3. Logistic regression analysis for risk factors of lower extremity amputation

\begin{tabular}{|c|c|c|}
\hline \multirow{2}{*}{ Variable } & \multicolumn{2}{|c|}{ Univariable analysis } \\
\hline & Odds ratio $(95 \% \mathrm{CI})$ & $p$ value \\
\hline Age & $1.01(0.98-1.03)$ & 0.607 \\
\hline Male sex & $1.13(0.63-2.05)$ & 0.677 \\
\hline Smoking & $0.84\left(0.45^{-1.59)}\right.$ & 0.595 \\
\hline Duration of DM & $1.00(0.97-1.03)$ & 0.929 \\
\hline Hypertension & $0.71(0.39-1.29)$ & 0.257 \\
\hline Prior amputation history & $1.22(0.46-3.25)$ & 0.694 \\
\hline $\mathrm{WBC} \geq 10, \times 10^{9} / \mathrm{L}$ & $1.92(1.07-3.44)$ & 0.030 \\
\hline $\mathrm{CRP} \geq 6, \mathrm{mg} / \mathrm{dL}$ & $3.46(1.83-6.53)$ & $<0.001$ \\
\hline eGFR & $1.00(0.10-1.01)$ & 0.409 \\
\hline Oral anti-diabetic medications & $1.34(0.70-2.56)$ & 0.384 \\
\hline Antiplatelet agents & $1.45(0.51-1.81)$ & 0.071 \\
\hline Statin & $0.79(0.41-1.52)$ & 0.485 \\
\hline PAD & $2.78(1.41-5.49)$ & 0.003 \\
\hline \multicolumn{3}{|l|}{ Wound size, $\mathrm{cm}^{2}$} \\
\hline$<10$ & 1.00 & \\
\hline 10 to $<25$ & $1.01(0.42-2.43)$ & 0.992 \\
\hline$\geq 25$ & $3.12(1.14-8.54)$ & 0.027 \\
\hline Bone or joint involvement of DFU & $4.11(2.04-8.29)$ & $<0.001$ \\
\hline Combined infection of DFU & $20.24(7.96-51.49)$ & $<0.001$ \\
\hline
\end{tabular}

CI, confidence interval; DM, diabetes mellitus; WBC, white blood cell; CRP, C-reactive protein; eGFR, estimated glomerular filtration rate; $\mathrm{PAD}$, peripheral artery disease; $\mathrm{DFU}$, diabetic foot ulcer.

mean $\mathrm{HbA1c}$ during whole follow-up period. The most common site for DFU was toe in both non-LEA and LEA group. While total prevalence of PAD was more prevalent in LEA patients, the total number of angioplasty procedures, including percutaneous ballooning and/or stenting and surgical bypass of peripheral artery during the whole follow-up period, was not significantly different in both groups. However, both infected ulcer and ulcer with bone or joint invasion were more frequently observed in LEA patients (Table 2).

We searched the risk factors for LEA (Table 3). After adjustment for age, smoking, presence of hypertension or $\mathrm{PAD}$, prior amputation history, eGFR, wound extent, combined infection, and bone or joint involvement of ulcer, multivariable logistic regression analysis results showed that combined PAD (OR, 4.39; 95\% CI, 1.33 to 14.55; $p=0.016)$, combined infection of DFU (OR, 11.39; 95\% CI, 2.55 to 50.93; $p=0.001$ ), and the depth (bone or joint involvement of ulcer) (OR, 3.74; 95\% CI, 1.10 to 12.70; $p=0.035)$ were significant prognostic factors in patients with type 2 diabetes who needed hospitalization due to DFUs (Table 4).

\section{DISCUSSION}

In this retrospective cohort study, we investigated the clinical outcome of DFUs in patients with type 2 diabetes who visited the Emergency Department or required hospitalization via outpatient clinic due to DFUs, and analyzed the data of those patients to illustrate several predictors of LEA.

DFU is a disastrous complication of diabetes, often leading to LEA. According to data from the National Hospital Discharge Survey, 28.4 LEA events occur per 10,000 patients with diabetes, and LEA among adults with diabetes was 10 times higher than for those without diabetes in 2010 [13]. 
Table 4. Logistic regression analysis for risk factors of lower extremity amputation

\begin{tabular}{|c|c|c|}
\hline \multirow{2}{*}{ Variable } & \multicolumn{2}{|c|}{ Multivariable analysis } \\
\hline & Odds ratio (95\% CI) & $p$ value \\
\hline Age & $1.01(0.96-1.06)$ & 0.798 \\
\hline Smoking & $0.76(0.19-3.03)$ & 0.694 \\
\hline Hypertension & $0.57(0.18-1.87)$ & 0.355 \\
\hline $\begin{array}{l}\text { Prior amputation } \\
\text { history }\end{array}$ & $2.03(0.33-12.53)$ & 0.445 \\
\hline eGFR & $1.00(0.99-1.01)$ & 0.915 \\
\hline PAD & $4.39(1.33-14.55)$ & 0.016 \\
\hline \multicolumn{3}{|l|}{ Wound size, $\mathrm{cm}^{2}$} \\
\hline$<10$ & 1.00 & \\
\hline 10 to $<25$ & $0.52(0.14-1.89)$ & 0.320 \\
\hline$\geq 25$ & $1.91(0.43-8.45)$ & 0.392 \\
\hline $\begin{array}{l}\text { Bone or joint } \\
\text { involvement of DFU }\end{array}$ & $3.74(1.10-12.70)$ & 0.035 \\
\hline $\begin{array}{l}\text { Combined infection } \\
\text { of DFU }\end{array}$ & $11.39(2.55-50.93)$ & 0.001 \\
\hline
\end{tabular}

CI, confidence interval; eGFR, estimated glomerular filtration rate; $\mathrm{PAD}$, peripheral artery disease; DFU, diabetic foot ulcer.

There have been many studies about the risk factors for DFU development; several risk factors for DFU have been reported, such as male sex, older age, history of previous ulcer, smoking, longer duration of diabetes, hypertension, neuropathy, PAD, deformity of foot, poor glycemic control, nephropathy, retinopathy, and nephropathy, etc. [14-17]. In contrast, there are not many longitudinal studies for prognosis of DFU have been reported. Several studies suggested the predictors of LEA such as older age, nephropathy, peripheral arterial disease, sensory neuropathy, uncontrolled DM, and infection [18]. However, there are still not many prospective cohort studies with a multicenter and relatively large sample size to evaluate the predictors of LEA at the present time.

After initial assessment of DFU, a treatment strategy should be established according to the proven risk factors and severity of each wound, as many clinical practice guidelines recommend [2]. Therefore, prognosis of DFU and predictors of LEA should be well understood [19].

The LEA rate (58.9\%) in this study was higher than those of other epidemiological studies of LEA for DFU (13.7\%) [17]. This can be explained by higher baseline severity of the DFU wound (according to University of Texas classification, 64 patients [33.3\%] were over class IIB), as many of our patients were admitted via emergency room for untreated chronic wound or referred from primary care for unhealed wound despite treatment. Lower limb amputation due to DFUs contributes to increased medical costs. Depending on hospitalization/non-hospitalization, diabetic foot amputation was associated with 1.6 or 2.1 times more annual days of healthcare use, and 2.0 or 2.3 times higher total medical cost than non-diabetic foot amputation. DFU was associated with 1.7 or 3.2 times higher total medical cost [6].

One of the important predictors for LEA in this study was depth of wound. On the basis of anatomy of foot and several classification system, we classified ulcers by depth: superficial soft tissue, fascia/muscle/tendon level, and bone or joint level [20]. Whether or not DFU invades the bone or joint level may be an especially important factor for prediction of prognosis. While several studies regarded the extent of the wound as an important factor related to LEA, it was not a significant predictor of LEA in our analysis [20]. This may suggest that decisions in the management of DFU could be dependent on the depth of wound, rather than extent of the wound.

Other predictors of LEA included the presence of PAD and the combined infection of ulcer. PAD is most easily detected by the ABI that is generally used. An angiography may reveal significant macrovascular disease requiring intervention. In this study, $\mathrm{PAD}$ was defined as $\mathrm{ABI} \leq 0.9$ or compatible findings of $\mathrm{PAD}$ in peripheral arterial angiography or CT. In terms of degree of ischemia, arterial oxygen supply can also be measured by transcutaneous oximetry. A transcutaneous oxygen tension higher than $30 \mathrm{mmHg}$ correlates with a high likelihood of wound healing. However, transcutaneous oxygen tension requires expensive equipment and a trained technician, and it cannot be routinely used [18]. Although there was more moderate to severe stenosis of arteries in LEA patients, there was not a statistically significant difference between patients with LEA and non-LEA.

A total of 76 patients among 192 DFU (39.6\%) had a percutaneous lower extremity angiography or lower extremity CT angiography at baseline examination. We 
classified the severity of stenosis of any arteries that perfused the DFU area as mild, moderate, and severe, which were confirmed by the expert radiologist or vascular surgeons. Although there was more moderate to severe stenosis of arteries in LEA patients, there was not a statistically significant difference between patients with LEA and non-LEA (Table 2). However, we believe that the statistical analysis for the characteristics of vascular stenosis would not be enough due to the small number of PAD evaluation.

There are many studies that suggest combined infection of DFU as a poor prognostic factor [21]. Therefore, pathogen-specific antibiotics therapy is one of the important factors for optimal treatment of DM foot infection, and should be included in the protocol for managing DFU in each hospital. There are several points to consider in the development of the protocol. First, the frequency and range of resistance to antibiotics are different in many regions [21]. Therefore, epidemiologic studies to investigate the pathogen that is the most common or the most virulent in each hospital are essential. Each pathogen's resistance to antibiotics also should be investigated. Second, proper identification techniques need to be introduced because the pathogens which are elucidated under the current culture-based techniques are not necessarily the most clinically important organisms [20].

The method used to elucidate the causative organisms in DFU was a wound discharge swab in this study. Therefore, cultured organisms include not only the true pathogen but also normal flora of the patient's skin. Some patients visited our hospital after several days of treatment with antibiotics. For that reason, organisms were not included in the analyses in this study.

Considering that important predictors for LEA in this study were the depth of wound and combined infection, for comprehensive management and improved wound care, a multidisciplinary team approach, including orthopedist, plastic surgeon, vascular surgeon, and infectionologist seems to be essential in the initial management of DFU $[8,19]$.

In general, adequate glycemic control is essential for prevention of DFUs and LEAs $[20,22]$. However, the mean $\mathrm{HbA1c}$ level during the follow-up period was not different between the LEA and non-LEA groups in this study. Glycemic control cannot be shown as important for DFU treatment due to the small sample size and retrospective design of our study. Metabolic correction, including high glucose, blood pressure, and lipid profile within target range, with prompt infection control, should be emphasized in DFU patients with type 2 diabetes.

There are several limitations in this study. First, due to the retrospective study design, we could not assess presence of diabetic neuropathy. Because most patients were referred from other primary care, available baseline data for diabetic complications was not sufficient, and additional examinations for neuropathy were not possible on severely damaged wounds. As the included patients were those who required admission, and the patients with too little available clinical data from the medical record were also excluded, selection bias has to be taken into account. Other limitations of this study include relatively small sample size, retrospective design, and single center studies. A larger, prospective study will be needed to validate the findings of the present investigation. Additionally, as many studies suggest that infection of DFU is an important predictor for LEA [21,23], the difference in organisms and antibiogram of each DFU should be identified in the future for proper treatment of DFU.

In conclusion, in cases of severe DFU requiring hospitalization, the LEA risk was high in patients with type 2 diabetes. Therefore, DFU prevention is essential. Routine examination of the feet is necessary during routine clinical practice. Whenever DFU is identified, prompt management of DFUs by a multidisciplinary team would play an important role in lowering the LEA rate.

\section{KEY MESSAGE}

1. In case of severe diabetic foot ulcer (DFU) enough to hospitalization, the lower extremity amputation (LEA) risk was high in patients with type 2 diabetes.

2. The depth of the wound, combined infection and peripheral artery disease were significant prognostic factor of LEA while the extent of the wound was not. Multidisciplinary team approach is important in management of DFU. 


\section{Conflict of interest}

No potential conflict of interest relevant to this article was reported.

\section{REFERENCES}

1. Centers for Disease Control and Prevention. National diabetes statistics report: estimates of diabetes and its burden in the United States, 2014 [Internet]. Atlanta (GA): U.S. Department of Health and Human Services, 2014 [cited 2017 Feb 16]. Available from: http://www.cdc.gov/diabetes.

2. Woodbury MG. Diabetic foot risk assessment. Diabetes Metab Res Rev 2016;32:376-378.

3. International Diabetes Federation. IDF Diabetes Atlas, 7 th ed [Internet]. Brussels (BE): International Diabetes Federation, 2015 [cited 2017 Feb 16]. Available from: http:// www.diabetesatlas.org.

4. Centers for Disease Control and Prevention. National health and nutrition examination survey, 2014 [Internet]. Cheongju (KR): National Health and Nutrition Examination Survey, 2014 [cited 2017 Feb 16]. Available from: https://knhanes.cdc.go.kr.

5. Amin N, Doupis J. Diabetic foot disease: from the evaluation of the "foot at risk" to the novel diabetic ulcer treatment modalities. World J Diabetes 2016;7:153-164.

6. Task Force Team for Basic Statistical Study of Korean Diabetes Mellitus of Korean Diabetes Association, Park IeB, Kim J, et al. Diabetes epidemics in Korea: reappraise nationwide survey of diabetes "diabetes in Korea 2007 ". Diabetes Metab J 2013;37:233-239.

7. Madanchi N, Tabatabaei-Malazy O, Pajouhi M, Heshmat R, Larijani B, Mohajeri-Tehrani MR. Who are diabetic foot patients? A descriptive study on 873 patients. J Diabetes Metab Disord 2013;12:36.

8. Hicks CW, Selvarajah S, Mathioudakis N, et al. Burden of infected diabetic foot ulcers on hospital admissions and costs. Ann Vasc Surg 2016;33:149-158.

9. Chung CH, Kim DJ, Kim J, et al. Current status of diabetic foot in Korean patients using national health insurance database. J Korean Diabetes Assoc 2006;30:372-376.

10. Levey AS, Bosch JP, Lewis JB, Greene T, Rogers N, Roth D. A more accurate method to estimate glomerular filtration rate from serum creatinine: a new prediction equation. Modification of Diet in Renal Disease Study Group. Ann Intern Med 1999;130:461-470.
11. Yun JS, Ko SH, Ko SH, et al. Cardiovascular disease predicts severe hypoglycemia in patients with type 2 diabetes. Diabetes Metab J 2015;39:498-506.

12. Bravo-Molina A, Linares-Palomino JP, Lozano-Alonso S, Asensio-Garcia R, Ros-Die E, Hernandez-Quero J. Influence of wound scores and microbiology on the outcome of the diabetic foot syndrome. J Diabetes Complications 2016;30:329-334.

13. Gregg EW, Li Y, Wang J, et al. Changes in diabetes-related complications in the United States, 1990-2010. N Engl J Med 2014;370:1514-1523.

14. Ahmad J. The diabetic foot. Diabetes Metab Syndr 2016;10:48-60.

15. Tomita M, Kabeya Y, Okisugi M, et al. Diabetic microangiopathy is an independent predictor of incident diabetic foot ulcer. J Diabetes Res 2016;2016:5938540.

16. Bruun C, Siersma V, Guassora AD, Holstein P, de Fine Olivarius N. Amputations and foot ulcers in patients newly diagnosed with type 2 diabetes mellitus and observed for 19 years: the role of age, gender and co-morbidity. Diabet Med 2013;30:964-972.

17. Parisi MC, Moura Neto A, Menezes FH, et al. Baseline characteristics and risk factors for ulcer, amputation and severe neuropathy in diabetic foot at risk: the BRAZUPA study. Diabetol Metab Syndr 2016;8:25.

18. Singh N, Armstrong DG, Lipsky BA. Preventing foot ulcers in patients with diabetes. JAMA 2005;293:217-228.

19. Hsu CR, Chang CC, Chen YT, Lin WN, Chen MY. Organization of wound healing services: the impact on lowering the diabetes foot amputation rate in a ten-year review and the importance of early debridement. Diabetes Res Clin Pract 2015;109:77-84.

20. Noor S, Zubair M, Ahmad J. Diabetic foot ulcer: a review on pathophysiology, classification and microbial etiology. Diabetes Metab Syndr 2015;9:192-199.

21. Uckay I, Aragon-Sanchez J, Lew D, Lipsky BA. Diabetic foot infections: what have we learned in the last 30 years? Int J Infect Dis 2015;40:81-91.

22. Beaney AJ, Nunney I, Gooday C, Dhatariya K. Factors determining the risk of diabetes foot amputations: a retrospective analysis of a tertiary diabetes foot care service. Diabetes Res Clin Pract 2016;114:69-74.

23. Quilici MT, Del Fiol Fde S, Vieira AE, Toledo MI. Risk factors for foot amputation in patients hospitalized for diabetic foot infection. J Diabetes Res 2016;2016:8931508. 